\title{
Edge Vortex Flow due to Inhomogeneous Ion Concentration
}

\author{
Hideyuki Sugioka* \\ Frontier Research Center, Canon Inc., 30-2, \\ Shimomaruko 3-chome, Ohta-ku, Tokyo 146-8501, Japan \\ and Department of Mechanical Systems Engineering, \\ Shinshu University, 4-17-1 Wakasato, Nagano 380-8553, Japan
}

(Dated: February 15, 2017)

\begin{abstract}
The ion distribution of an open parallel electrode system is not known even though it is often used to measure the electrical characteristics of an electrolyte. Thus, for an open electrode system, we perform a non-steady direct multiphysics simulation based on the coupled Poisson-Nernst-Planck and Stokes equations and find that inhomogeneous ion concentrations at edges cause vortex flows and suppress the anomalous increase in the ion concentration near the electrodes. A surprising aspect of our findings is that the large vortex flows at the edges approximately maintain the ion-conserving condition, and thus the ion distribution of an open electrode system can be approximated by the solution of a closed electrode system that considers the ion-conserving condition rather than the Gouy-Chapman solution, which neglects the ion-conserving condition. We believe that our findings make a significant contribution to the understanding of surface science.
\end{abstract}

PACS numbers: 47.57.-s, 85.90.+h, 82.45.-h, 83.50.Lh

\footnotetext{
*E-mail: hsugioka@shinshu-u.ac.jp
} 
Introduction: The Poisson-Nernst-Planck (PNP) equation is well known as a fundamental equation in the field of electrokinetic phenomena and it reveals various ion diffusion phenomena in an electrolyte [1]. In our previous study, we found the exact solution of the one-dimensional (1D) steady PNP equation by using the ion-conserving Poisson-Boltzmann (ICPB) theory, which considers an ion-conserving condition with the Poisson-Boltzmann (PB) equation [2]. However, in practice, a pair of finite-length parallel open electrodes in a microfluidic channel filled with an electrolyte are often used to measure the electrical characteristics of the electrolyte. For this open electrode configuration, some researchers may consider that the widely used classical Gouy-Chapman (GC) solution [3, 4], which neglects the ion-conserving condition, is more valid than the ICPB solution because ions can be introduced through the open configuration. Thus, it is important to clarify the physics of the ion diffusion phenomena of this finite electrode system. In particular, it is important to understand the ion concentration near finite-length electrodes in an open configuration to interpret the results of an electric measurement.

However, to the best of our knowledge, the phenomena of a finite electrode system have not been yet in depth. Therefore, in this paper, we focus on the two-dimensional (2D) time evolution simulation of the ion diffusion phenomena of a finite parallel electrode system in the presence of a dc electric field as a first step. In particular, we will show that the inhomogeneous ion concentration at the edges of the electrodes causes large vortex flows around the edges and that these flows suppress the anomalous increase in the ion concentration according to the GC solution, and thus substantially maintain the ion-conserving condition.

Theory: Figure 1 shows the 2D model system used in our direct simulation [5, 6] and the mechanism of the generation of edge vortex flows. As shown in Fig. 1, we analyze ion diffusion phenomena between finite-length parallel blocking electrodes separated by distance $w$ (e.g., $20 \mu \mathrm{m}$ ) under a dc applied voltage $V_{0}$ in a microfluidic channel (or chamber) filled with an electrolyte. Here, $L(=2 w)$ is the length of the channel (chamber) and $L_{0}(=w)$ is the length of each electrode. As shown in the figure, the large ion concentration near the electrodes causes a flow from the inside region to the outside region, and thus macroscopic vortex flows are generated at the edges. Furthermore, the method of the 2D direct simulation is the same as that in our previous study $[5,6]$ and one can find detailed explanations in Refs. $[5,6]$; however, we briefly explain the method here for the convenience of readers. Namely, by using the finite element method (FEM) with the finite volume method (FVM), 


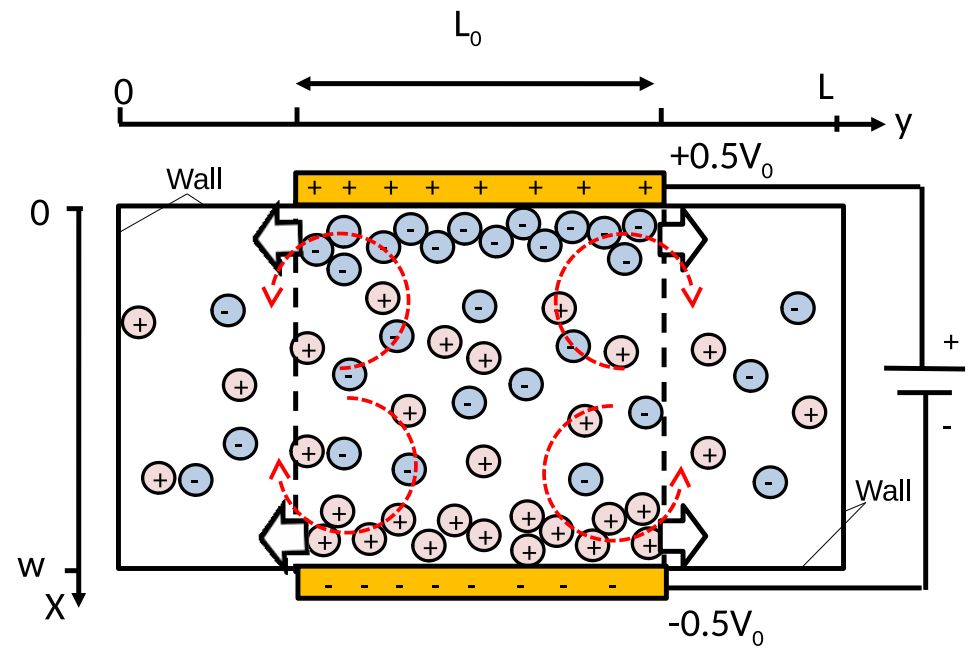

FIG. 1. (Color online) 2D model system used in our direct simulation and mechanism of the generation of large vortex flows at the edges. As shown in the figure, the large ion concentration near the electrodes causes a flow from the inside region to the outside region and thus macroscopic vortex flows are generated at the edges. Here, $V_{0}$ is the applied voltage, $w($ e.g., $20 \mu \mathrm{m}$ ) is the distance between the blocking electrodes, $L(=2 w)$ is the length of the channel (chamber), and $L_{0}$ $(=w)$ is the length of each electrode.

we perform the $2 \mathrm{D}$ direct simulation using the Poisson equation $\left(\varepsilon \nabla^{2} \Phi+\rho_{e}=0\right)$, NernstPlanck equation $\left(\frac{\partial C_{ \pm}}{\partial t}+\nabla \cdot\left[-D\left(\nabla C_{ \pm} \pm \frac{z e}{k T} C_{ \pm} \nabla \Phi\right)+C_{ \pm} \boldsymbol{u}\right]=0\right)$, and Stokes equation ( $\left.-\nabla p+\mu \nabla^{2} \boldsymbol{u}-\rho_{e} \nabla \Phi=0, \nabla \cdot \boldsymbol{u}=0\right)$. Here, $\varepsilon\left(=80 \varepsilon_{0}\right)$ is the dielectric permittivity, $\varepsilon_{0}$ is the vacuum permittivity, $\rho_{e}$ is the charge density, $C_{+}\left(C_{-}\right)$is the positive (negative) ion concentration, $\Phi$ is the potential, $t$ is time, $D\left(\sim 10^{-9} \mathrm{~m}^{2} / \mathrm{s}\right)$ is the ion diffusivity, $k$ is the Boltzmann constant, $T$ is the temperature, ze is the ion charge, $\boldsymbol{u}$ is the flow velocity, $p$ is pressure, and $\mu(\sim 1 \mathrm{mPa} \cdot \mathrm{s})$ is the viscosity. Note that we use the nondimensional formulations in Ref. [2] in our calculations. Thus, all results are a function of $\kappa=w / \lambda_{D}$, $v_{0}=V_{0} / V_{c}, \tau=t / T_{0}$, and $F_{0}=\frac{\epsilon}{2 \mu D}\left(\frac{k T}{e z}\right)^{2} \kappa^{2}$, where $\lambda_{D}$ (e.g., $1 \mu \mathrm{m}$ for water at $\mathrm{pH}=7$; see Sec. VIII-F in Ref. [2] for detail) is the Debye screening length, $V_{c}=k T / e z=25 \mathrm{mV}$ is the reference voltage, and $T_{0}=w^{2} / D$ is the reference time. Also, the reference flow velocity is denoted as $U_{c}=D / w$. Namely, the specific dimensional values are just an example, for 


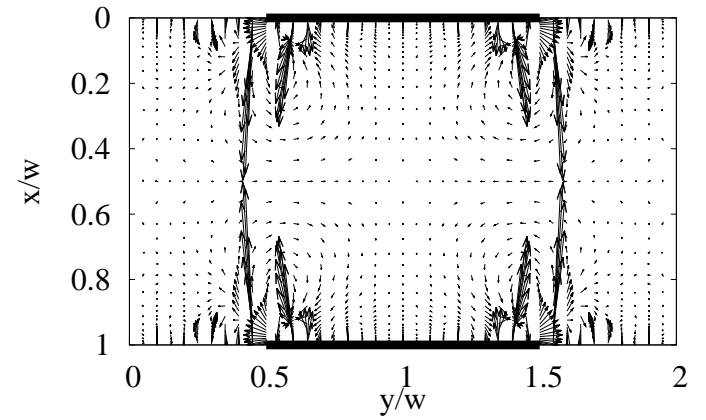

(a) Steady strong edge flow: Flow field at

$$
t / T_{0}=0.499 \text { with } f=0.2 .
$$

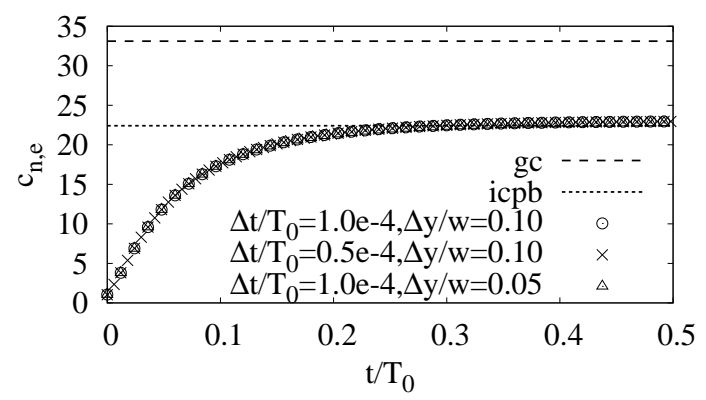

(c) Dependence of $c_{n, e}$ on $t$.

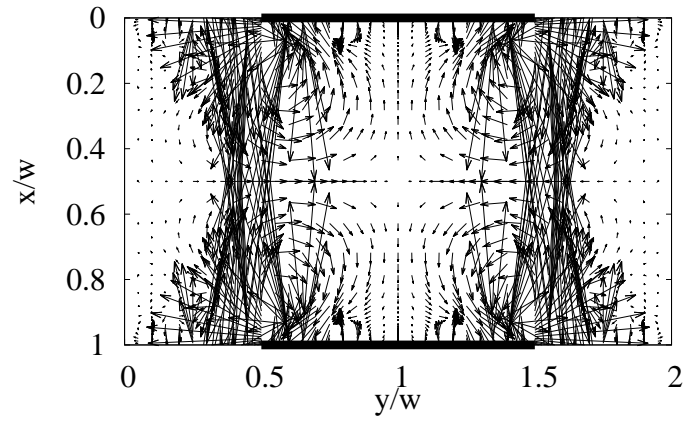

(b) Steady edge vortex flow: Flow field at $t / T_{0}=0.499$ with $f=1.0$.

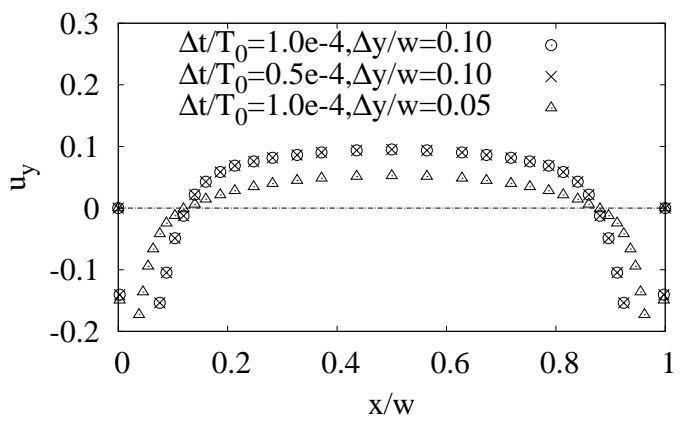

(d) Dependence of $u_{y}$ on $x / w$ at $y / w=0.5$

$$
\left(t / T_{0}=0.499\right)
$$

FIG. 2. Steady edge vortex flow and related characteristics. Here, $v_{0}=7, \kappa=w / \lambda_{D}=20$, and $F_{0}=99.77$, for example, when $w=20 \mu \mathrm{m}$ and $D=10^{-9} \mathrm{~m}^{2} / \mathrm{s}, U_{c}=D / w=50 \mu \mathrm{m} / \mathrm{s}$ and $T_{0}=w^{2} / D=0.4 \mathrm{~s}$.

example, when $w=20 \mu \mathrm{m}, \lambda_{D}=1 \mu \mathrm{m}$, and $D=10^{-9} \mathrm{~m}^{2} / \mathrm{s}, \kappa=20, T_{0}=0.4 \mathrm{~s}$, and $U_{c}=50 \mu \mathrm{m} / \mathrm{s}$. Furthermore, as boundary conditions we consider that $\Phi= \pm 0.5$ at $x / w=0$ and 1 , and $\boldsymbol{u}=0$ at the walls and the electrodes with the blocking condition; i.e., the ions cannot pass through the electrode-electrolyte boundary.

Results: Figure 2 shows the steady edge vortex flow and related characteristics for $v_{0}=$ $V_{0} / V_{c}=7, \kappa=W / \lambda_{D}=20$, and $F_{0}=99.77$. As shown in Fig. 2(a), we can observe strong edge flows mainly from the inside region to the outside region above the edges at $t / T_{0}=0.499$ with $f=1.0$, where $f$ is the magnification factor of the vector length used in the visualization. In Fig. 2(b), we can also observe four vortex flows at the edges at $t / T_{0}=0.499$ with $f=0.2$. Note that we increased the magnification factor $f$ from 0.2 to 1.0 to observe the flow field in detail. Figure 2(c) shows the dependence of $c_{n, e}=C_{n, e} / C_{0}$ on $t$, where $C_{0}$ 
is the bulk ion concentration and $C_{n, e}$, is the negative ion concentration of the calculation cell adjacent to the center of the top positive electrode. Since $c_{n, e}$ becomes constant at $t / T_{0}=0.499$ in Fig. 2(c), we can recognize that the edge vortex flow at $t / T_{0}=0.499$ is in the steady state. In Fig. 2(c), symbols show the numerical results, whereas dotted and broken lines show the ICPB and GC solutions of the steady state, respectively. Namely, we find that the ion concentration adjacent to the electrode in the steady state is (at least) approximately described by the ICPB solution rather than the GC solution. In Fig. 2(c) [and also, Figs. 2(d) and 4(c)], circles, crosses, and triangles show the numerical results at $\left(\Delta t / T_{0}, \Delta y / w\right)=(0.00010,0.10),(0.00005,0.10)$, and $(0.00010,0.05)$, respectively, where $\Delta t$ is the time interval and $\Delta y$ is the space interval in the $y$ direction. Thus, from Fig. 2(c), we find that the value of $c_{n, e}$ converges at $\left(\Delta t / T_{0}, \Delta y / w\right)=(0.00010,0.10)$ because it does not change for a change in $\left(\Delta t / T_{0}, \Delta y / w\right)$. Therefore, our numerical results concerning $c_{n, e}$ are reliable. Note that we use an inhomogeneous mesh that is symmetrical about the $x=0.5$ plane in the $x$ direction and the $i$ th space interval is described as $\Delta x_{i} / w=\Delta x_{0} r^{i-1}$, where $\Delta x_{0}=0.1 \lambda_{D}$ is the initial space interval and $r=1.3$ is the ratio of the increase. Here, the inhomogeneous mesh with $\Delta x_{0}=0.1 \lambda_{D}$ and $r=1.3$ is suitable for the ion diffusion problem, since the calculation using the mesh was verified by detailed examination by comparing the numerical results with the exact solutions in Ref. [2]; thus, our calculations concerning $c_{n, e}$ are reliable.

However, Fig. 2(d) shows that the dependence of $u_{y}=U_{y} / U_{c}$ on $x / w$ (at $y / w=0.5$ and $\left.t / T_{0}=0.499\right)$, where $U_{y}$ is the flow velocity in the $y$ direction, does not converge under the condition; and thus we may need to use a more refined mesh in the future to improve our numerical calculations. Nevertheless, Fig. 2(d) clearly shows the characteristics of outward and inward flows due to the vortex flow above the edge position of the electrodes; i.e., it clearly indicates that the ions of the electric double layer near the electrode in the electrode region are drained by an outward flow on the order of $0.1 U_{c}=5 \mu \mathrm{m} / \mathrm{s}$, whereas the ions of the outside region are introduced into the electrode region by an inward flow on the order of $0.05 U_{c}=2.5 \mu \mathrm{m} / \mathrm{s}$ in the bulk area. Note that since the ion concentration near the electrode is much higher than that in the bulk region, the drained and introduced ions are balanced even though the area of the curves below the line $u_{y}=0$ is smaller than that above the line $u_{y}=0$ in Fig. 2(d). Furthermore, although the convergence of $u_{y}$ in Fig. 2(d) is unsatisfactory, the drained and introduced ions are balanced, probably because 


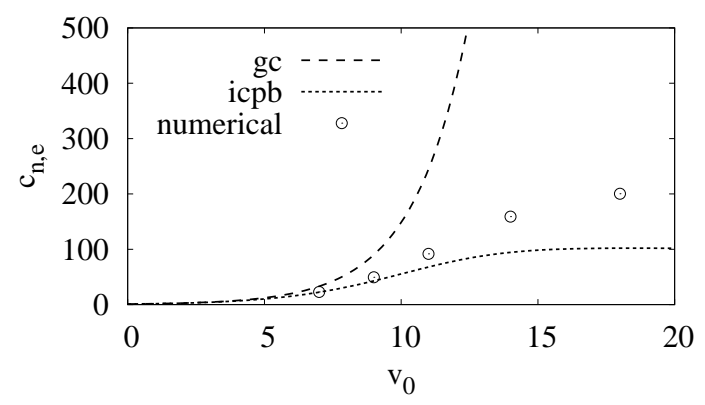

FIG. 3. Steady ion concentration near electrodes of the open blocking electrode system showing dependence of $c_{n, e}$ on $v_{0}$ at $t / T_{0}=0.499$. Here, $v_{0}=7, \kappa=w / \lambda_{D}=20$, and $F_{0}=99.77$, for example, when $w=20 \mu \mathrm{m}$ and $D=10^{-9} \mathrm{~m}^{2} / \mathrm{s}, U_{c}=D / w=50 \mu \mathrm{m} / \mathrm{s}$ and $T_{0}=w^{2} / D=0.4$ s. Circles show the numerical results of the open blocking electrode system, whereas dotted and broken lines show the ICPB and GC solutions, respectively, in the steady state.

of the continuous characteristics of the vortex flow for the incompressible fluid. Thus, our calculations using the condition $\left(\Delta t / T_{0}, \Delta y / w\right)=(0.00010,0.10)$ can be used to discuss the ion concentrations of the open electrode system, although the condition should be further improved in the future.

Figure 3 shows the dependence of $c_{n, e}$ on $v_{0}$ at $t / T_{0}=0.499$. As shown in Fig. 3 , the steady ion concentration near the electrodes of the open blocking system is roughly approximated by the ICPB solution rather than the GC solution. Specifically, the ICPB solution very accurately predicts $c_{n, e}$ for the open electrode system for $v_{0} \leq 9$; then, the accuracy of the prediction gradually decreases as $v_{0}$ increases; and the prediction of the ICPB is half the numerical value at $v_{0}=18$. Although the ICPB solution cannot predict the behavior of $c_{n, e}$ completely, from Fig. 3, we can recognize that the ICPB solution is a much better approximation of the ion concentration of the open electrode system than the GC solution at $v_{0}>10$ (i.e., $V_{0}>250 \mathrm{mV}$ ).

Figure 4 shows the reverse vortex flow in a non-steady state. As shown in Fig. 4(a), we can observe four transient reverse vortex flows at the edges at $t / T_{0}=0.01$. This is partially because the crowding of the high ion concentration occurs transiently near the edges, as shown in Fig 4(b), and thus the flows in the $\pm y$ directions at the edges are disturbed. Another reason for the reverse flow is that it is probably much easier to take ions from the outside region near the edge than from the bulk area of the electrode region 


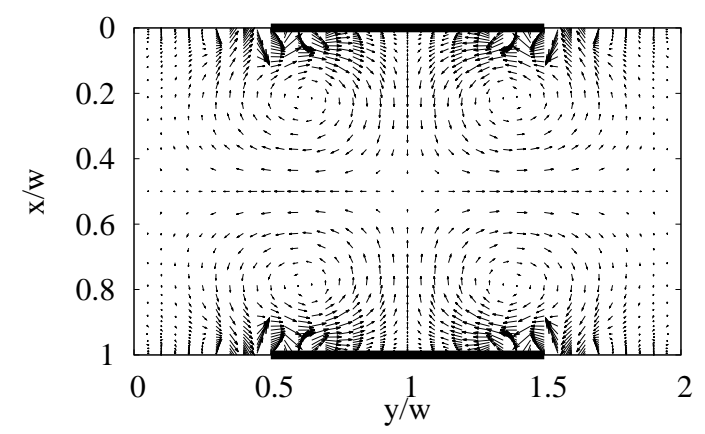

(a) Reverse vortex flow $(f=1.0)$

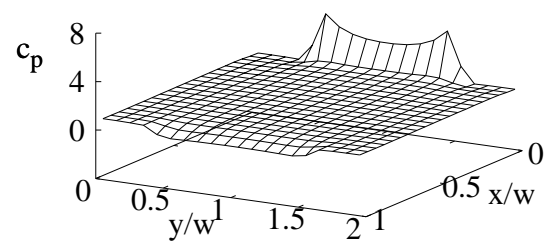

(b) Distribution of $c_{p}$

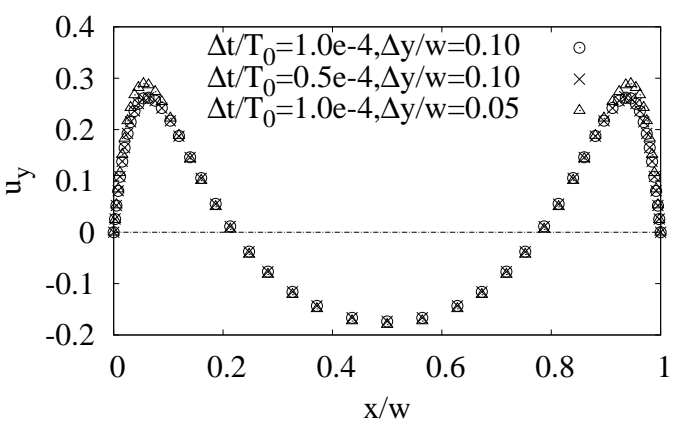

(c) Dependence of $u_{y}$ on $x / w$ at $y / w=0.5$

FIG. 4. Reverse vortex flow in a non-steady state at $t / T_{0}=0.010$. Here, $v_{0}=7, \kappa=w / \lambda_{D}=20$, and $F_{0}=99.77$, for example, when $w=20 \mu \mathrm{m}$ and $D=10^{-9} \mathrm{~m}^{2} / \mathrm{s}, U_{c}=D / w=50 \mu \mathrm{m} / \mathrm{s}$ and $T_{0}=w^{2} / D=0.4 \mathrm{~s}$. In (c), symbols show the numerical results.

(i.e., $0.5 \leq y / w \leq 1.5$ ); in other words, the electrodes strongly attract ions from the nearest surrounding area, particularly when the ions have not yet been attracted. Figure 4(c) shows the dependence of $u_{y}=U_{y} / U_{c}$ on $x / w$ at $y / w=0.5$ and $t / T_{0}=0.010$. In contrast to $u_{y}$ in Fig. 2(c), $u_{y}$ in Fig. 4(c) almost converges at $t / T_{0}=0.010$ under the conditions, and thus our calculations for $u_{y}$ are reliable at $t / T_{0}=0.010$. Furthermore, from Fig. 4(c), we 


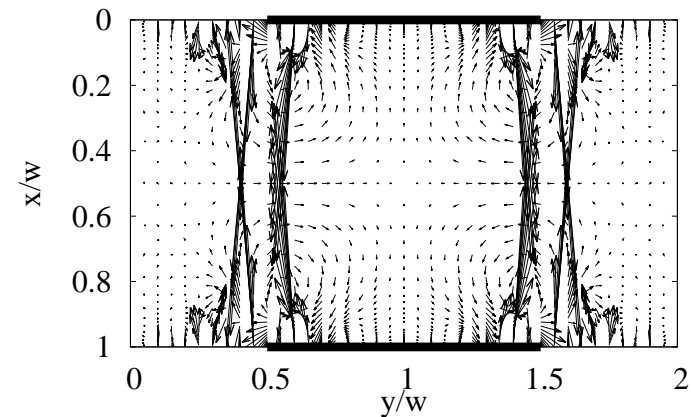

(a) Flow field at $t / T_{0}=0.499$ with $f=1.0$

$$
\left(\lambda_{s} / w=0.01\right) .
$$

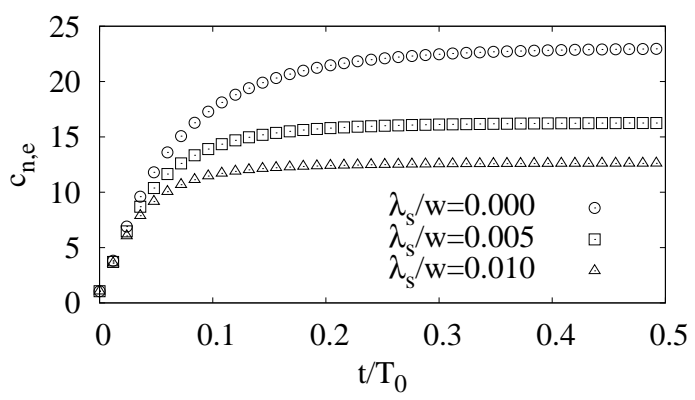

(c) Dependence of $c_{n, e}$ on $t$.

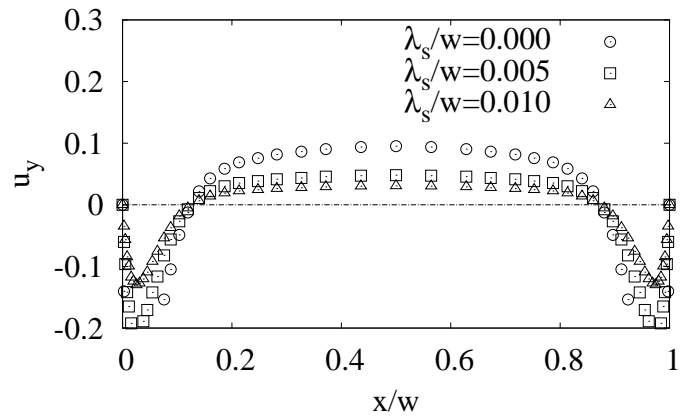

(b) Dependence of $u_{y}$ on $x / w$ at $y / w=0.5$

$$
\left(t / T_{0}=0.499\right)
$$

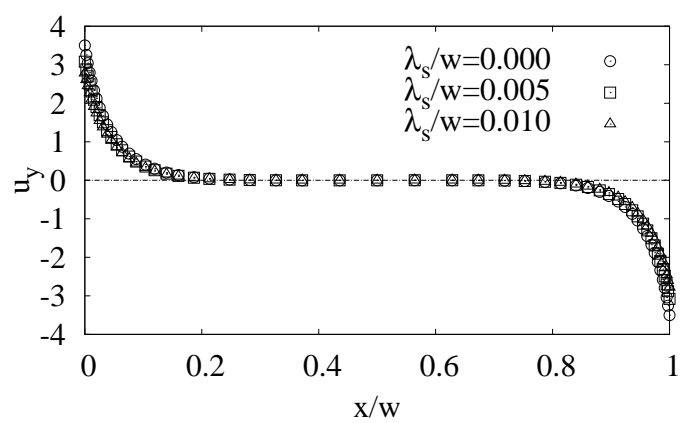

(d) Dependence of $\phi$ on $x / w$ at $y / w=1.0$

$$
\left(t / T_{0}=0.499\right)
$$

FIG. 5. Steady edge vortex flow and related characteristics considering a Stern layer. Here, $\Delta t / T_{0}=10^{-4}$ and $\Delta y / w=0.1 ; v_{0}=7, \kappa=w / \lambda_{D}=20$, and $F_{0}=99.77$, for example, when $w=20 \mu \mathrm{m}$ and $D=10^{-9} \mathrm{~m}^{2} / \mathrm{s}, U_{c}=D / w=50 \mu \mathrm{m} / \mathrm{s}$ and $T_{0}=w^{2} / D=0.4 \mathrm{~s}$.

find that an inward flow of approximately $0.25 U_{c}(12.5 \mu \mathrm{m} / \mathrm{s})$ is generated near the edge, whereas an outward flow of approximately $0.18 U_{c}(9 \mu \mathrm{m} / \mathrm{s})$ is generated in the bulk area.

For simplicity, we neglect the existence of a Stern layer in the calculations shown in Figs. 2-4. However, a Stern layer always exists on electrodes in a real system. Thus, in Fig. 5, we perform calculations considering a Stern layer. Namely, Fig. 5 shows the steady edge vortex flow and related characteristics considering a Stern layer with the Robin boundary condition on the electrodes [1] for $v_{0}=V_{0} / V_{c}=7, \kappa=W / \lambda_{D}=20$, and $F_{0}=99.77$. From Fig. 5, we can recognize that edge vortex flows can be generated in real ion systems, not only in an ideal system that neglects a Stern layer. Specifically, as shown in Fig. 5(a), we can also observe edge flows at $t / T_{0}=0.499$ with $f=1.0$, although the flow velocity is slightly lower than that in Fig. 2(b) because of the existence of the Stern layer. In fact, 
as shown in Fig. 5(b), $u_{y}$ decreases as $\lambda_{s}$ increases, where $\lambda_{s}$ is the thickness of the Stern layer. This is also because $c_{n, e}=C_{n, e} / C_{0}$ decreases as $\lambda_{s}$ increases, as shown in Fig. $5(\mathrm{c})$. In other words, the Stern layer simply reduces the substantial applied voltages (i.e., $|\phi|$ at $x / w= \pm 1$ ) slightly as shown in Fig. $5(\mathrm{~d})$.

Discussion: (1) Inplications of our findings: Our direct simulations clarified that the open parallel electrode configuration causes a large edge vortex flow at the edges due to the inhomogeneous ion concentration. The predicted flow velocities of 0.5 to $12.5 \mu \mathrm{m} / \mathrm{s}$ at $v_{0}=7$ (i.e., $V_{0}=175 \mathrm{mV}$ ) are relatively small; however, they can still be detected by a careful experiment. Thus, the prediction of edge vortex flows should be verified experimentally in the future. Furthermore, although the GC solutions may be valid for ordinary classical experiments for $v_{0}<10$, we clarified that the ion concentration near the open blocking electrodes for $v_{0}>10$ can be much more accurately estimated by the ICPB solution, which considers the ion-conserving condition, than the GC solution, which allows the unlimited introduction of ions. Namely, because of the existence of the steady vortex flow, the infinite introduction of the ions, which is considered to occur are believed by many researchers, is naturally suppressed. Instead, since the introduced ions are expected to be pushed out by the edge vortex flow of the incompressible fluid, the ion-conserving conditions are almost maintained and thus the ICPB (i.e., PNP) solution is approximately justified, even for the open electrode system.

(2) Source of energy sustaining the vortex flow in the steady state: Although this is obvious, the source of energy sustaining the vortex flow in the steady state is the dc power supply (in Fig. 1) connected to the blocking electrodes. However, some researchers may argue that without using Faradaic reactions and ac voltages, it is not clear how the energy is supplied and how the viscous dissipation is compensated. Here, for the half region of $x \leq 0.5$, we consider a simple CR electric circuit model consisting of an electric-double-layer capacitor $C_{D} \simeq \epsilon S / \lambda_{D}$ and a bulk resistor $R_{b}$ [1], where $S$ is the area of the electrode. In the case of a closed electrode system, it should be universally agreed that by connecting a de power supply of voltage $V_{0}^{\prime}=V_{0} / 2$ to the electrode, the capacitor is charged and the accumulated energy of the capacitor is described by $U_{D}=C_{D} V_{0}^{\prime 2} / 2=Q_{D}^{2} / 2 C_{D}$, where $Q_{D}=C_{D} V_{0}^{\prime}$ is the accumulated charge. Of course, the electric energy is supplied from the power supply and the transfer of the electric energy is usually considered to stop when the potential difference of the capacitor $V_{D}$ becomes equal to $V_{0}^{\prime}$ under the implicit assumption that there is no 
dissipation in the system. However, if there is a dissipation in the real system, $U_{D}, V_{D}$, and $Q_{D}$ decrease but are rapidly recovered by the energy transferred from the power supply. This is the meaning of the boundary condition of $\Phi=V_{0}^{\prime}$ at $x / w=0$ in the numerical calculation. Specifically, there is dissipation due to the outward flow of the double-layer charge in our open electrode system but it is rapidly compensated by the energy transferred from the power supply. In other words, because of the dissipation of the real system, we may consider that a vortex current exists at the electrodes in the real system; however, the ideal limit of such phenomena is numerically described by the boundary condition of $\Phi=V_{0}^{\prime}$ at $x / w=0$, and we need not consider the vortex current in our calculations. This answers the question of how the energy is supplied and how the viscous dissipation is compensated without using Faradaic reactions and ac voltages.

(3) Reason for ignoring the finite-size effect of ions: The edge vortex flow tends to take place more easily as the ion concentration near the electrodes increases. Thus, if we examine an edge vortex flow with a low concentration of ions near the electrodes, we can also expect that the observed phenomena can occur even with a high concentration of ions. Thus, in this study, we consider water of $\mathrm{pH}=7$ (i.e., $C_{0}=10^{-7} \mathrm{~mol} / \mathrm{l}$ ) with a suitable separation distance $w(=20)$ as the ideal low-concentration limit in a typical microfluidic configuration. As a result, we need not consider the finite-size effect of ions because the ion concentration near the electrodes is approximately $200 C_{0}$ for $v_{0}=20$ as shown in Fig. 3. In other words, since the concentration of water molecules is approximately $55.6 \mathrm{~mol} / \mathrm{l}$, we need not consider the finite-size effect of ions provided $C_{0}<55.6 / 200 \simeq 0.278 \mathrm{~mol} / \mathrm{l}$. Namely, our calculations that predict an edge vortex flow are justified for a wide range of ion concentrations (i.e., $10^{-7} \leq C_{0}<55.6 / 200 \simeq 0.278 \mathrm{~mol} / \mathrm{l}$ ), although the finite-size effect of ions becomes important as $w$ becomes large [7]. This is the main reason why we ignore the finite-size effect of ions with a low concentration near electrodes as a first step.

Conclusion: By performing a 2D direct simulation, we have shown that a pair of finitelength parallel electrodes in a channel causes a steady vortex flow near the edge owing to an inhomogeneous large ion concentration under dc applied voltages and that the vortex flow significantly suppresses the increase in the steady ion concentration near the electrodes and thus approximately maintains the ion-conserving condition. Therefore, even for the open electrode configuration, the ICPB solution is much more accurate than the GC solution for estimating the ion distribution between electrodes. 


\section{ACKNOWLEDGMENTS}

This work was partially supported by JSPS KAKENHI Grant Number JP16K05650.

[1] M. Z. Bazant, K. Thornton, and A. Ajdari, Phys. Rev. E 70, 021506 (2004).

[2] H. Sugioka, Phys. Rev. E 86, 016318 (2012).

[3] G. Gouy, J. Phys. 9, 45 (1910).

[4] D. L. Chapman, Philos. Mag. 25, 475 (1913).

[5] H. Sugioka, Phys. Rev. E 90, 013007 (2014).

[6] H. Sugioka, Phys. Rev. E 94, 022609 (2016).

[7] H. Sugioka, Advances in Colloid and Interface Science 226, Part A, 44 (2015). 